\title{
Cuban Adolescents Requesting Presymptomatic Testing for Spinocerebellar Ataxia Type 2
}

\author{
Tania Cruz-Mariño, ${ }^{1}$ Luis Velázquez-Pérez, ${ }^{2}$ Yanetza González-Zaldivar, ${ }^{3}$ \\ Raúl Aguilera-Rodríguez, ${ }^{4}$ Miguel Velázquez-Santos, ${ }^{1}$ Yaimé Vázquez-Mojena, ${ }^{3}$ \\ Annelié Estupiñán-Rodríguez, ${ }^{4}$ José Miguel Laffita-Mesa, ${ }^{3}$ Rubén Reynaldo-Armiñán, ${ }^{5}$ \\ Luis Enrique Almaguer-Mederos, ${ }^{3}$ and Milena Paneque ${ }^{6}$ \\ ${ }^{1}$ Predictive Genetics Department, Center for the Research and Rehabilitation of Hereditary Ataxias, Reparto Edecio Pérez, \\ CP 80100 Holguín, Cuba \\ ${ }^{2}$ Neurophysiology Department, Center for the Research and Rehabilitation of Hereditary Ataxias, Reparto Edecio Pérez, \\ CP 80100 Holguín, Cuba \\ ${ }^{3}$ Molecular Biology Department, Center for the Research and Rehabilitation of Hereditary Ataxias, Reparto Edecio Pérez, \\ CP 80100 Holguín, Cuba \\ ${ }^{4}$ Clinical Department, Center for the Research and Rehabilitation of Hereditary Ataxias, Reparto Edecio Pérez, \\ CP 80100 Holguín, Cuba \\ ${ }^{5}$ International Relationships Department, Medical University of Holguín, CP 80100 Holguín, Cuba \\ ${ }^{6}$ Center for Predictive and Preventive Genetics IBMC, Institute for Molecular and Cell Biology, University of Porto, \\ Rua do Campo Alegre 823, 4150-180 Porto, Portugal
}

Correspondence should be addressed to Tania Cruz-Mariño; taniacruz@hotmail.ca and Luis Velázquez-Pérez; velazq63@yahoo.com

Received 23 March 2013; Accepted 12 April 2013

Academic Editors: D. Radojkovic and W. Wang

Copyright (C) 2013 Tania Cruz-Mariño et al. This is an open access article distributed under the Creative Commons Attribution License, which permits unrestricted use, distribution, and reproduction in any medium, provided the original work is properly cited.

Since 2001 a program for the presymptomatic testing of families affected with SCA2 has been under development in Cuba. According to the initial protocol, access would be given to nonsymptomatic individuals at $50 \%$ risk, over 18 years; nevertheless, eleven minors requested their inclusion in the program. A retrospective and descriptive study based on the analysis of the medical records belonging to these individuals was designed. Being aware of how challenging clinical settings of predictive genetic testing for minors are, this paper reviews their profile, the outcome of the carried out studies, as well as the reproductive option chosen by the gene positive consultands. The mean age at the time of testing was 16.2 years. Nine adolescents completed the protocol (three had positive test results) and two withdrew. They had a distinctive profile; all were females, pregnant, motivated by the risk assessment for their descendants, and interested in PND with termination of the gestation were the fetus a carrier. Nevertheless, once the result of the test is known, the gene positive consultands chose discordant reproductive options. Further research is necessary to assess the long-term psychological impact in both gene positive and gene negative participants, as well as in their parents and at-risk relatives.

\section{Introduction}

In February 2001, health professionals in Cuba designed a protocol for genetic counseling, presymptomatic testing
(PST), and prenatal diagnosis (PND) of families affected with spinocerebellar ataxia type 2 (SCA2) $[1,2]$.

With an autosomal dominant mode of inheritance, SCA2 is considered a late onset neurodegenerative disease mainly 
characterized by cerebellar dysfunction (ataxic gait, cerebellar dysarthria, dysmethria, dysdiadochokinesia) associated with slow saccades, severe tremor of postural or action type, peripheral neuropathy, cognitive disorders, and other multisystemic features $[3,4]$.

The disease in question raises in this country the highest worldwide prevalence ( 6.57 cases per $10^{5}$ inhabitants) with 753 affected individuals belonging to 200 unrelated families and 7000 at-risk descendants [5], who contribute to the high uptake rates of the SCA2 PST (24.91\%) [6].

According to the SCA2 PST initial protocol, access would be given to nonsymptomatic individuals at $50 \%$ risk, over 18 years, who did not have severe psychiatric disturbances. The SCA2 mutation must have been identified in at least a relative $[1,2]$. Nevertheless, some at-risk individuals under 18 years requested their inclusion in the PST program along these years (2001-2012).

Testing minors for adult-onset diseases is mainly justified by its direct benefit through either medical intervention or preventive measures. Most guidelines recommend postponing testing, because there is fear that testing could create devastating social, emotional, psychosocial, and educational consequences [7]. However, it is necessary to allow space for the utilization of judgment by genetics professionals about whether genetic testing is in the child's best interests [8].

Some authors consider that clinicians performing such tests may feel vulnerable in sharing the outcomes, given that such testing is contrary to current recommendations [9]. Others advocate to collect empirical evidence in controlled and clearly defined settings and to reform guidelines based on this evidence, since predictive genetic testing in minors has become a reality [10].

This paper reviews the profile of the Cuban adolescents who requested the SCA2 PST, the outcome of the carried out studies, as well as the reproductive option chosen by the gene positive consultands.

\section{Materials and Methods}

A retrospective and descriptive study was designed, based on the analysis of the medical records belonging to eleven teenagers who requested their inclusion in the PST program between February 2001 and December 2012.

The predictive protocol used during the period of study has been published elsewhere $[1,2]$. It included at least two genetic counseling sessions, a neurological examination, and psychological screening/diagnostic evaluations prior to genetic testing (in order to estimate whether consultands were able to handle results). In addition to the psychological test batteries, an anamnesis guide was used to obtain sociodemographic data, psychological antecedents, and personal history.

Psychological followup evaluations were offered one week, four weeks, six months, and one year after genetic testing results disclosure [1].

DNA testing was based on the detection of an abnormal CAG trinucleotide repeat expansion in the ATXN2 gene through fragment analysis [11]. Alleles up to 31 CAG repeats are normal, alleles with 32 repeats or larger are expanded, while alleles from 35 to 500 CAG are fully penetrant [11-16]. At disclosure, the individuals were informed of both alleles' sizes.

All participants were informed about PST procedures and protocol, as well as the possibility of using information from their clinical records in clinical research, and they gave their separate written consent for both. The study was approved by the Institutional Ethics Committee.

\section{Results}

Eleven female teenagers requested their inclusion in the PST program, all of them pregnant and having the assessment of the risk for their descendants as their main motivation for taking the test. They all expressed their intention to perform PND in the case they were carriers, with termination of the gestation if the fetus was a carrier.

The established protocol of consultations was followed in all cases and psychological attention was reinforced. Additional consultations were performed in order to discuss personal pathological antecedents at length, ego strengths, coping strategies, social support available, and familial issues. Degree of maturity, voluntariness, and adequate understanding of information were considered crucial criteria in the assessment of these adolescents.

The mean age at the time of testing was 16.2 years (range 13-17 years, sd 1.33 years). One case was 13 years old; her parents took the initiative of involving her in PST, but she consented to the test. The rest of the cases were self-referring, one of them was 16, two cases were 15, and the seven left were 17 (Figure 1).

Nine participants were married or living in common law $(81.82 \%)$, while two were single $(18.18 \%)$. Seven individuals had a secondary school level (63.64\%), and only four had finished the preuniversitary school (36.36\%). Most of them were nonreligious (81.82\%).

Nine out of eleven teenagers (81.82\%) completed the protocol, while two withdrew after the second genetic counseling session (18.18\%); both were 17 years old and referred fear of being unable to cope with an unfavorable result as the reason for withdrawal.

Among those individuals who completed the protocol, three had positive test results (33.33\%): the 13-year-old minor and two 17-year-old adolescents.

The 13-year old consultand was a major concern for our group. Her short age aside, her parents did not agree to the pregnancy and were pushing her to terminate it. She insisted that she would make a decision once she knew her genetic status. During the disclosure session of her positive result (genotype 22/40) she remained calmed and expressed that she was prepared for the obtained result and that she had decided the early termination of pregnancy.

On the contrary, one of the other gene positive adolescents (genotype 22/34) decided to carry the pregnancy to term without testing the fetus, while the other one (genotype 22/43) requested her inclusion in the PND, but a fetal death occurred before the amniocentesis could be performed. 


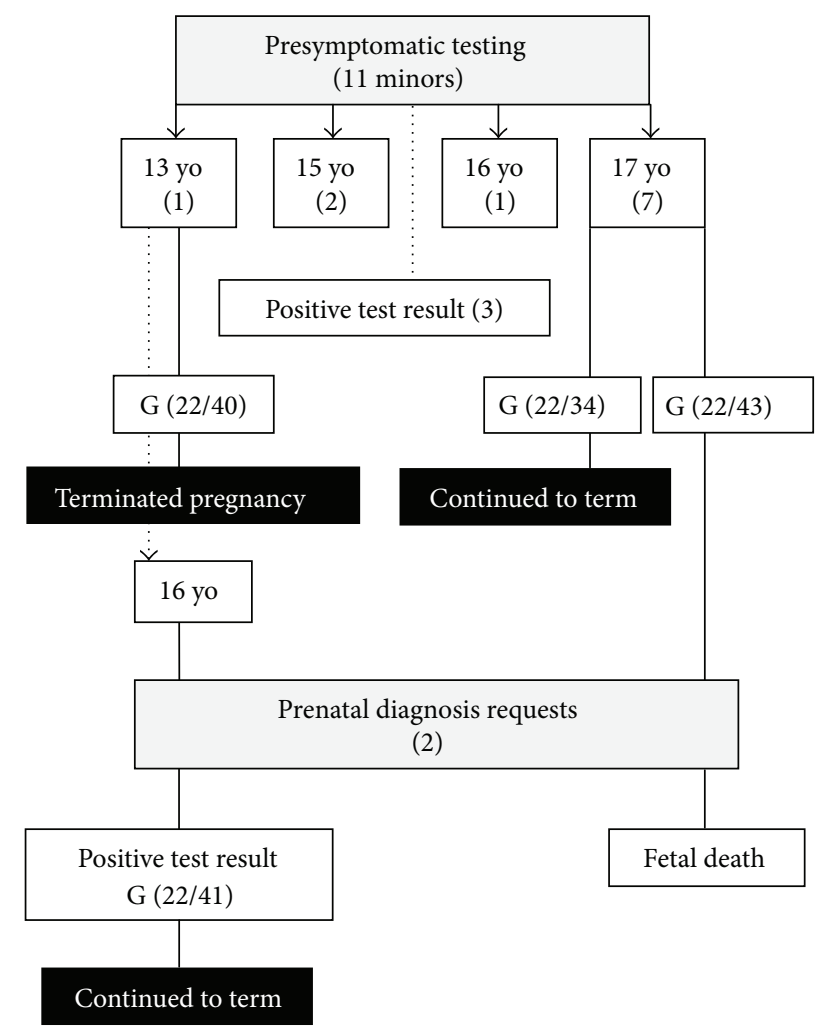

FIGURE 1: Flowchart showing the outcome of the SCA2 presymptomatic testing in eleven minors and the reproductive option chosen by the gene positive consultands. yo: years old. G: genotype.

Three years later, the former 13-year old minor and now 16 years old adolescent returned pregnant and requested the PND. Her fetus resulted positive to the test (genotype 22/41), nonetheless she decided to carry the pregnancy to term.

\section{Discussion}

To offer predictive genetic testing to young people for adultonset conditions where no treatment or preventive intervention exists is a controversial subject submitted to intense scientific debate. There is international consensus about the fact that where there are no "urgent medical reasons," predictive testing should be postponed until a child is able to give his/her own consent as a competent adolescent or as an adult $[8-10,17,18]$.

In $\mathrm{HD}$ it is recommended that the minimum age of testing be age of majority [19]. For SCAs there are no advantages for minors either; therefore, PST should not be undertaken [18].

Particularly, the group of eleven teenagers included in our PST program requested the test in the context of an ongoing pregnancy. The risk assessment for their descendants was their main motivation to take the test; similarly, this was the main reason for adult individuals in Cuba to participate in the SCA2 PST [6].

In addition to the adolescent unplanned pregnancy disadvantages and the psychological consequences of this type of testing, for this SCA2 at-risk population we had to weigh the lack of access to predictive testing programs due to their short age and the potential risks and benefits of their almost simultaneous inclusion in PST and PND.

Guidelines should always be assessed in the particular local legal context and cannot always be translated to another cultural setting [7]. Determining whether genetic testing should be recommended or discouraged in any particular case requires both an ethical and a pragmatic assessment of the unique circumstances of each patient [20]. Therefore, every single case requesting to participate in our program was discussed among the members of the multidisciplinary team in the predictive genetics department (clinical geneticist, neurologist, genetic counselor, psychologist, psychiatrist, and social worker). The results of such analysis were then presented to the ethical committee of the institution accompanied by the request letter signed by the minors and their parents. The fact that these consultands were already coupled and requesting PND as a reproductive option was a powerful reason to include them in the program. Since they were capable of choosing testing, requested the test by themselves, and appeared to understand the implications of the potential results, the genetic testing would then provide them with the psychological benefit of relieving anxiety [20].

Furthermore, the line between adult-onset conditions and conditions that cause problems in childhood is not always clear [20]. In our population 111 minors under 18 years have been clinically affected by SCA2, among them $52(46,85 \%)$ have deceased due to this condition; this is why for many Cuban families SCA2 is not an adult-onset disease anymore.

In presymptomatic subjects, approximately 15 years before the onset of ataxia the slowing of horizontal saccades at $60^{\circ}$ of target displacement is present as the earliest subclinical sign, followed by the reduction of REM sleep percentage with decreased rapid eye movements' density, decrease of sensory amplitudes, increased P40 latency, motor performance deficits demonstrated through the prism adaptation task, and reduced capabilities to identify odors in a smell identification test [21].

Moreover, a severe phenotype in six children with massive expansions of ATXN2 gene has been described elsewhere [22].

All the teenagers who requested their inclusion in the PST program were females. It is in accordance with a female predominance among adults requesting the SCA2 PST $(59,77 \%)$ [6]. At the same time, it suggests that at-risk male teenagers are either not interested in PST or deal less frequently with unplanned/unwanted pregnancies and their consequences. Another possible explanation is the great sense of responsibility of women respecting reproductive issues, as it has been described for general higher female PST uptake $[1,2]$. We believe it is possible that some other male teenagers can be facing paternity without seeking counseling support.

All the cases but one were self-referring, their voluntariness suggesting competence and maturity. The 13-yearold case was well informed about her genetic risk, had an adequate understanding of the test and its potential implications, received appropriate counselling, and showed the capacity to make her own decisions; all these are required 
conditions to consider a minor as competent to undergo genetic testing $[23,24]$.

Apart from this case, it is our empirical experience along 12 years that, in our population, parents do not request the inclusion of their children in the SCA2 PST; they understand and agree the importance of postponing the test till their descendants are 18 years old. Something different takes place in other populations where genetic services are often confronted with parental requests to determine carrier status of healthy children [25].

The rate of withdrawal among teenagers was similar to the one reported for adults taking part in the SCA2 PST program (19.43\%) [6]. From our perspective, that an adolescent decides to withdraw after receiving genetic counseling because she understands that she is not able to cope with the result should be considered a success.

Despite the fact that all the teenagers were pregnant, only one among three gene positive testees requested the PND. One possibility for this is the very short period for psychological adjustment to the carrier diagnosis, having to decide immediately about PND. Again, the decision to refrain from genetic testing or avoid learning its results can involve the same degree of autonomy as the decision to undertake testing and embrace its outcome [26].

The decision to carry the pregnancy of a gene positive fetus to term is not privative of the adolescent here presented; it has been also the decision of $28.57 \%$ of the older pregnant women in our SCA2 PND program (7 out of 27) [27]. The ethical, legal, and psychosocial implications that the nontermination of these pregnancies brings about have been extensively discussed [28-31].

The unplanned adolescent pregnancy is undoubtedly the main problem underlying the self-referral of teenagers requesting PST. Genetic counseling practice at the community level is an appropriate setting for the preconception care focusing on SCA2 at-risk adolescents.

The small size of the sample constitutes a limitation of the present study, together with the lack of similar reports which would allow the comparison of our results.

\section{Conclusions}

Predictive testing protocols are tributaries of continued supervision and must be adequated through a case-by-case approach; some aspects like inclusion criteria in the case of minors deserve especial attention. We felt the need to adapt, as we have been doing, the national protocol, considering the characteristics of the at-risk population in our country and also their cultural values and familial issues, as it is expected in an effective practice of genetic counseling; this is why inclusion criteria have been expanded to reach SCA2 at-risk teenagers with interest in PND.

The Cuban adolescents requesting the PST had a distinctive profile; they were all females, pregnant, motivated by the risk assessment for their descendants, and interested in PND. Nevertheless, once the result of the test is known, the gene positive consultands chose discordant reproductive options.
Further research is necessary to assess the long-term psychological impact in both gene positive and gene negative participants, as well as in their parents and at-risk relatives.

Are identity changes in noncarrier teenagers so beneficial as we thought? Can they continue life planning without the shadow of the disease? Are they really aware of full implications of this testing experience? The potential benefits and/or harmful effects of testing must be investigated over time.

We would like to invite international groups to discuss these particular issues if this is the case that PST is being requested by pregnant adolescents in their everyday practice.

\section{Conflict of Interests}

The authors declare no conflict of interests.

\section{Acknowledgments}

The authors are deeply indebted to the SCA2 Cuban families that participated in the PST program and to the Cuban Ministry of Health. They are thankful to José Luis Guisao Martínez for the language corrections.

\section{References}

[1] H. M. Paneque, A. L. Prieto, R. R. Reynaldo et al., "Psychological aspects of presymptomatic diagnosis of spinocerebellar ataxia type 2 in Cuba," Community Genetics, vol. 10, no. 3, pp. 132-139, 2007.

[2] M. Paneque, C. Lemos, K. Escalona et al., "Psychological followup of presymptomatic genetic testing for spinocerebellar ataxia type 2 (SCA2) in Cuba," Journal of Genetic Counseling, vol. 16, no. 4, pp. 469-479, 2007.

[3] L. Velázquez-Pérez, R. Rodríguez-Labrada, J. C. GarcíaRodríguez, L. E. Almaguer-Mederos, T. Cruz-Mariño, and J. M. Laffita-Mesa, "A comprehensive review of spinocerebellar ataxia type 2 in Cuba," Cerebellum, vol. 10, no. 2, pp. 184-198, 2011.

[4] J. J. Magaña, L. Velázquez-Pérez, and B. Cisneros, "Spinocerebellar ataxia type 2: clinical presentation, molecular mechanisms, and therapeutic perspectives," Molecular Neurobiology, vol. 47, no. 1, pp. 90-104, 2013.

[5] L. Velázquez Pérez, G. S. Cruz, N. Santos Falcón, E. Almaguer Mederos, K. Escalona Batallán, R. Rodríguez Labrada et al., "Molecular epidemiology of spinocerebellar ataxias in Cuba: insights into SCA2 founder effect in Holguín," Neuroscience Letters, vol. 454, no. 2, pp. 157-160, 2009.

[6] T. Cruz-Mariño, L. Velázquez-Pérez, Y. González Zaldivar, R. Aguilera-Rodríguez, M. Velázquez-Santos, Y. Vázquez-Mojena et al., "The cuban program for predictive testing of SCA2: 11 years and 768 individuals to learn from," Clinical Genetics, 2013.

[7] P. Borry, L. Stultiens, H. Nys, J. J. Cassiman, and K. Dierickx, "Presymptomatic and predictive genetic testing in minors: a systematic review of guidelines and position papers," Clinical Genetics, vol. 70, no. 5, pp. 374-381, 2006.

[8] M. Parker, "Genetic testing in children and young people," Familial Cancer, vol. 9, no. 1, pp. 15-18, 2010.

[9] R. E. Duncan and M. B. Delatycki, "Predictive genetic testing in young people for adult-onset conditions: where is the empirical evidence?" Clinical Genetics, vol. 69, no. 1, pp. 8-16, 2006. 
[10] C. Mand, L. Gillam, M. B. Delatycki, and R. E. Duncan, "Predictive genetic testing in minors for late-onset conditions: a chronological and analytical review of the ethical arguments," Journal of Medical Ethics, vol. 38, no. 9, pp. 519-524, 2012.

[11] J. M. Laffita-Mesa, L. C. Velázquez-Pérez, N. Santos-Falcon, T. Cruz-Mariño, Y. González-Zaldívar, Y. Vázquez-Mojena et al., "Unexpanded and intermediate CAG polymorphisms at the SCA2 locus (ATXN2) in the Cuban population: evidence about the origin of expanded SCA2 alleles," European Journal of Human Genetics, vol. 20, no. 1, pp. 41-49, 2012.

[12] G. Imbert, F. Saudou, G. Yvert et al., "Cloning of the gene for spinooerebellar ataxia 2 reveals a locus with high sensitivity to expanded CAG/glutamine repeats," Nature Genetics, vol. 14, no. 3, pp. 285-291, 1996.

[13] K. Sanpei, H. Takano, S. Igarashi et al., "Identification of the spinocerebellar ataxia type 2 gene using a direct identification of repeat expansion and cloning technique, DIRECT," Nature Genetics, vol. 14, no. 3, pp. 277-284, 1996.

[14] S. M. Pulst, A. Nechiporuk, T. Nechiporuk et al., "Moderate expansion of a normally biallelic trinucleotide repeat in spinooerebellar ataxia type," Nature Genetics, vol. 14, no. 3, pp. 269-276, 1996.

[15] S. Choudhry, M. Mukerji, A. K. Srivastava, S. Jain, and S. K. Brahmachari, "CAG repeat instability at SCA2 locus: anchoring CAA interruptions and linked single nucleotide polymorphisms," Human Molecular Genetics, vol. 10, no. 21, pp. 24372446, 2001.

[16] J. Sequeiros, S. Seneca, and J. Martindale, "Consensus and controversies in best practices for molecular genetic testing of spinocerebellar ataxias," European Journal of Human Genetics, vol. 18, no. 11, pp. 1188-1195, 2010.

[17] P. Borry, G. Evers-Kiebooms, M. C. Cornel, A. Clarke, and K. Dierickx, "Genetic testing in asymptomatic minors: background considerations towards ESHG Recommendations," European Journal of Human Genetics, vol. 17, no. 6, pp. 711-719, 2009.

[18] J. Sequeiros, J. Martindale, and S. Seneca, "EMQN best practice guidelines for molecular genetic testing of SCAs," European Journal of Human Genetics, vol. 18, no. 11, pp. 1173-1176, 2010.

[19] R. MacLeod, A. Tibben, M. Frontali et al., "Recommendations for the predictive genetic test in Huntington's disease," Clinical Genetics, vol. 83, no. 3, pp. 221-231, 2013.

[20] E. C. Caga-anan, L. Smith, R. R. Sharp, and J. D. Lantos, “Testing children for adult-onset genetic diseases," Pediatrics, vol. 129, no. 1, pp. 163-167, 2012.

[21] L. Velázquez-Pérez, R. Rodríguez-Labrada, H. J. Freund, and G. Auburger, "Spinocerebellar Ataxia type 2," in Spinocerebellar Ataxia, J. Gazulla, Ed., pp. 77-102, 2012.

[22] A. R. Paciorkowski, Y. Shafrir, J. Hrivnak, M. C. Patterson, M. B. Tennison, H. B. Clark et al., "Massive expansion of SCA2 with autonomic dysfunction, retinitis pigmentosa, and infantile spasms," Neurology, vol. 77, no. 11, pp. 1055-1060, 2011.

[23] P. Tozzo, L. Caenazzo, and Daniele Rodriguez, "Genetic testing for minors: comparison between Italian and British guidelines," Genetics Research International, vol. 2012, Article ID 786930, 4 pages, 2012.

[24] European Society of Human Genetics, "Genetic testing in asymptomatic minors: recommendations of the European Society of Human Genetics," European Journal of Human Genetics, vol. 17, no. 6, pp. 720-721, 2009.

[25] P. Borry, T. Goffin, H. Nys, and K. Dierickx, "Attitudes regarding carrier testing in incompetent children: a survey of European clinical geneticists," European Journal of Human Genetics, vol. 15, no. 12, pp. 1211-1217, 2007.

[26] S. Fox, "Adolescents, graduated autonomy, and genetic testing," Genetics Research International, vol. 2012, Article ID 946032, 6 pages, 2012.

[27] T. Cruz-Mariño, L. Velázquez-Pérez, Y. González Zaldivar, R. Aguilera-Rodríguez, M. Velázquez-Santos, Y. Vázquez-Mojena et al., "Couples at risk for spinocerebellar ataxia type 2: the cuban prenatal diagnosis experience," Community Genetics. In press.

[28] A. Clarke, D. Fielding, L. Kerzin-Storrar et al., "The genetic testing of children," Journal of Medical Genetics, vol. 31, no. 10, pp. 785-797, 1994.

[29] S. A. Simpson, M. W. Zoeteweij, K. Nys et al., "Prenatal testing for huntington's disease: a European collaborative study," European Journal of Human Genetics, vol. 10, no. 11, pp. 689-693, 2002.

[30] S. Creighton, E. W. Almqvist, D. MacGregor, B. Fernandez, H. Hogg, J. Beis et al., "Predictive, pre-natal and diagnostic genetic testing for Huntington's disease: the experience in Canada from 1987 to 2000," Clinical Genetics, vol. 63, no. 6, pp. 462-475, 2003.

[31] F. H. Richards and G. Rea, "Reproductive decision making before and after predictive testing for Huntington's disease: an Australian perspective," Clinical Genetics, vol. 67, no. 5, pp. 404411, 2005. 

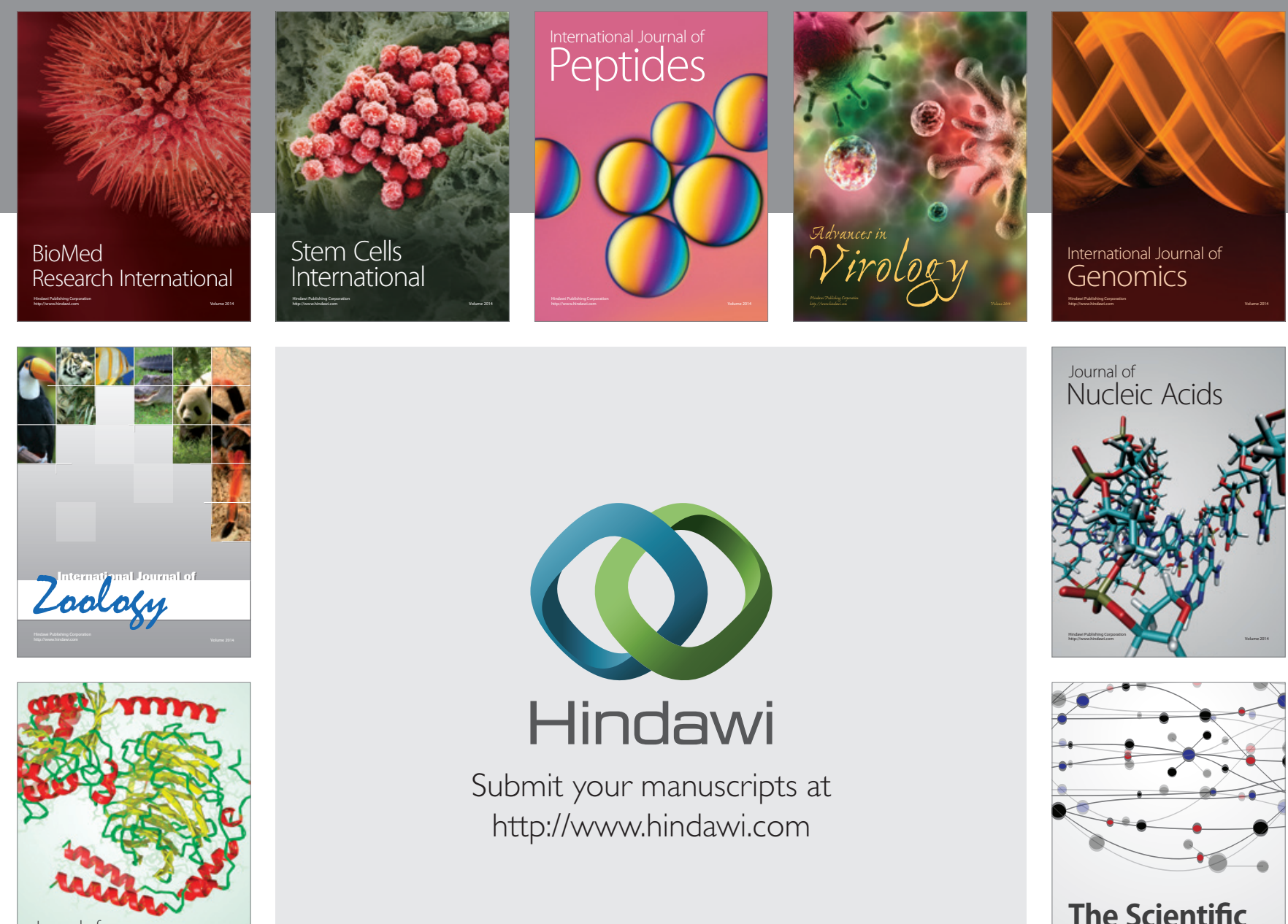

Submit your manuscripts at

http://www.hindawi.com

Journal of
Signal Transduction
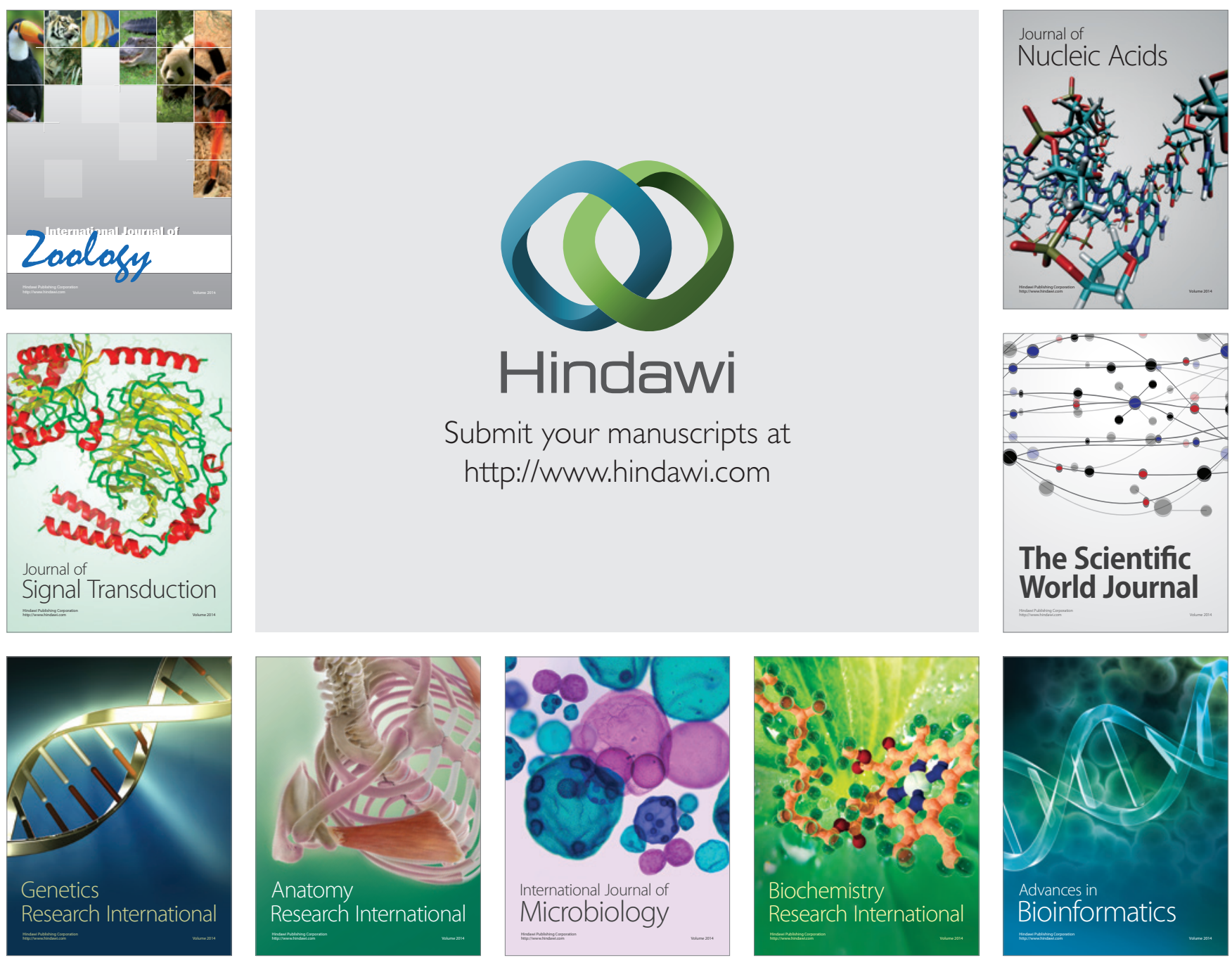

The Scientific World Journal
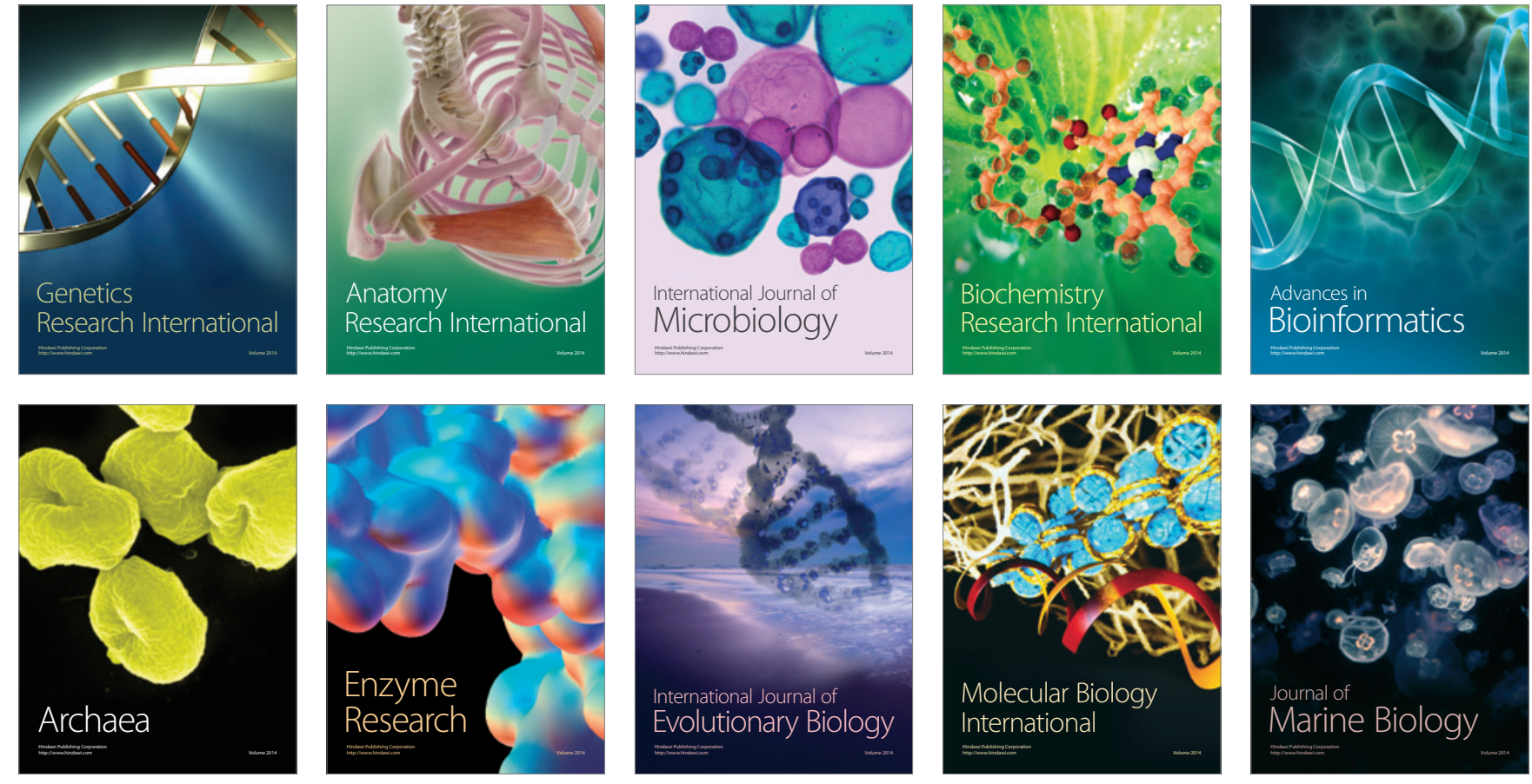